\title{
Oscillations in a nonlinear spring mechanism with one degree of freedom
}

\author{
Leonid Kondratenko and Lubov Mironova* \\ Moscow Aviation Institute (State National Research University), Moscow, Russia
}

\begin{abstract}
A new method for studying dynamics in a nonlinear springloaded mechanism is described, which consists in describing a spring as an elastic solid rod with conditional density and elastic modulus. When studying vibrations in such a rod, instead of the well-known second-order differential equation, two derived first-order equations are used that relate the acceleration and gradient of speed to the velocity and gradient of the change in the voltage of the particles of a given body. The frequency characteristic of the linear mechanism is given. The behavior of the mechanism in the presence of significant nonlinearity is considered. The self-oscillation parameters are determined that make it possible to estimate the design life.
\end{abstract}

\section{Introduction}

Devices with spring-loaded parts are used in many mechanisms. In many cases, these devices can be considered as two-link mechanical systems containing input and output links, interconnected by an elastic line. The input link is given the movement that is transmitted by the output device to the actuator. Moreover, the output link, connected by an elastic line, must make reciprocating movements, that is, return the output mechanism to its original position with a given accuracy. Despite the fact that these mechanisms are simple in design and convenient to operate, when transmitting motion, longitudinal or torsional vibrations occur in them, which lead to a deviation from the given law of motion or the exact positioning of the output link. In this case, we should talk about the stability of the dynamic system [1-4]. Loss of stability leads to self-oscillations.

In the time of analyzing operation of the considered mechanisms are usually investigated displacements. However, during fluctuations, especially forced ones, which are often the main mode of operation, it is important to know not only the features of movement, but also the laws of stress change [5], which have a significant impact on the efficiency of work of the mechanism.

\section{Design scheme and major applied developments}

Consider a device (Fig. 1), where a leading rod (pusher), moving at a speed of $v_{1}$ moves along the $x$ axis a body of mass $m$ located in the guides, and the vibrations at the driven end

\footnotetext{
*Corresponding author: mironova_lub@mail.ru
} 
do not affect the movement of the pusher. The resistance force $F$ and the friction force $F_{m}=$ $h \mathrm{v}_{2}$ also act on the body, where $\mathrm{h}$ is the coefficient of friction losses proportional to the speed of the movement body.

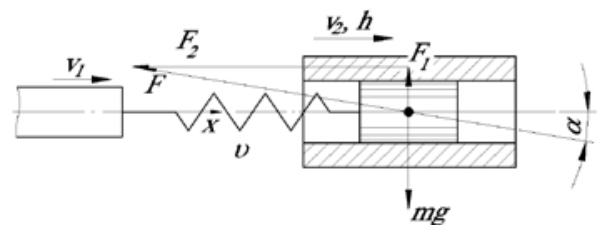

Fig. 1. Scheme of a spring-loaded mechanism.

Suppose that in static the spring has the characteristic $\Delta x C=F_{0}$, where $\Delta x$ is the movement; $C$ is the stiffness coefficient; $F_{0}$ is power. We rewrite the given relation in the form

$$
\Delta x=\frac{l F_{0}}{f E_{y}}, f=\frac{\pi\left(D^{2}-D_{1}^{2}\right)}{4}, E_{y}=\frac{l C}{f} .
$$

Here $l$ is the length of the spring, $f$ is the cross-sectional area of the spring; $E_{y}$ is conditional modulus of elasticity of the spring; $D, D_{1}$ are outer and inner diameters of the spring.

It is known that under the action of longitudinal loads in a twisting wire with a diameter $\mathrm{d}$ of the spring, tangential stresses arise. Taking into account various distortions, the maximum tangential stresses are determined by the relation [6]

$$
\tau_{\max }=K \frac{8 D_{0} F}{\pi d^{3}} .
$$

Here $D_{0}$ is the average diameter of the spring by the centers of the circumferences of the wire; $K$ is a coefficient taking into account the curvature of the coil and the influence of shear force.

If a spring is considered as a rod with section $f$, then under the action of a longitudinal force, conditional compressive or tensile stresses will develop in it

$$
\sigma_{y}=\frac{\Delta x E_{y}}{l}=K_{1} \tau_{\max } .
$$

Here, the coefficient $K_{1}$ characterizes the geometric parameters of the spring mechanism.

We assume that the spring operates within elasticity, and after the preload is applied by some static force $F_{0}$, on the body acts the variable component of the small amplitude force $F_{a}$. Under such conditions, the conditional density of the spring material $\rho_{y}$ can be considered constant and equal to

$$
\rho_{y}=k_{n} \rho_{m} \frac{l_{1} d^{2}}{\left(D^{2}-D_{1}^{2}\right) l}
$$

where $\rho_{m}$ is the actual density of the wire material; $k_{n}$ is coefficient taking into account the change in the diameter of the wire at the ends of the spring $l_{1}$ is the length of the wire.

Under these assumptions, in the framework of the one-dimensional model, the wellknown equation of longitudinal elastic vibrations for displacements can be written in the form [7] 


$$
\frac{\partial^{2} u}{\partial t^{2}}=\frac{E_{y}}{\rho_{y}} \frac{\partial^{2} u}{\partial x^{2}} .
$$

We supplement equation (5) by the equation quantity of momentum in the differential form [7-9], by taking $v=\partial u / \partial t$. We obtain

$$
\rho_{y} \frac{\partial v}{\partial t}=-\frac{\partial \sigma_{y}}{\partial x} .
$$

From formulas (5) and (6) follows the equation

$$
E_{y} \frac{\partial^{2} u}{\partial x^{2}}=-\frac{\partial \sigma_{y}}{\partial x} .
$$

After differentiating (7) with respect to the coordinate $x$ and integrating with respect to $t$, we obtain

$$
E_{y} \frac{\partial v}{\partial x}=-\frac{\partial \sigma_{y}}{\partial t} .
$$

The derived equations (6) and (8) describe the elastic vibrations in the spring relatively fully, and they are similar to the equations obtained for solid metal rods $[7,10,11]$.

As a result of the transition under zero initial conditions and the one-dimensional Laplace transform, we obtain the equations

$$
\rho_{y} s \cup(s)=-\frac{d \sigma_{y}(s)}{d x} ; E_{y} \frac{d \cup(s)}{d x}=-s \sigma_{y}(s) .
$$

Here $s$ is a complex variable, $s=j \omega ; \cup(s), \sigma_{y}(s)$ are the Laplace images of functions $v(t)$ and $\sigma_{y}(t)$, respectively; $\omega$ is the circular frequency.

The solutions of equations (9) have the form [7]

$$
\begin{gathered}
\sigma_{y}(s, x)=\sigma_{1 y}(s, 0) \operatorname{ch}\left[\theta_{n}(s) x\right]-\frac{\theta_{n}(s)}{s} E_{y} \mathrm{v}_{1} \operatorname{sh}\left[\theta_{n}(s) x\right] . \\
u(s, x)=v_{1}(s, 0) \operatorname{ch}\left[\theta_{n}(s) x\right]-\frac{s}{\theta_{n}(s) E_{n}} \sigma_{1 y}(s, 0) \operatorname{sh}\left[\theta_{n}(s) x\right] . \\
\theta_{n}(s)= \pm \sqrt{s\left[\rho_{y} s+\psi(s)\right] / E_{y}} .
\end{gathered}
$$

Here $\theta_{n}(s)$ is the operator coefficient wave propagation; $\psi$ is a function that takes into account friction losses during the movement of spring coils.

It should be noted that the internal friction $\psi$ in a solid rod is due to the dependence of the elastic modulus $E$ on the vibration frequency [12]. On the spring can, will an additional effect appear which remains to be determined. In subsequent discussions, we neglect this friction and take

$$
\theta(s)= \pm s \sqrt{\rho_{y} / E_{y}}
$$


The functions $\sigma_{y}(s, x), \cup(s, x)$ describe the fluctuations in the speeds of motion and stresses in the elastic power line.

Assuming that the momentum from the pusher to the body is transmitted completely, i.e. there are no reflected waves, the friction of the turns is insignificant, and we obtain the equation of motion in the Laplace images in the form [7]

$$
\mathrm{\cup}_{2}(s)\left[1-c+h_{n} \vartheta_{n}(s) s+m \vartheta_{n}(s) s^{2}\right]=\frac{1-c}{c h[\theta(s) l]} \mathrm{\cup}_{1}(s)-F(s) \vartheta_{n}(s) s
$$

and the equation the fluctuations of the conditional stress

$$
\begin{gathered}
\sigma_{y}(s)=\frac{1}{f(1-c)\left[1-c+h_{n} \vartheta_{n}(s) s+m \vartheta_{n}(s) s^{2}\right]} \times \\
\times\left[F(s)+\frac{\cup_{1}(s)\left(h_{n}+m s\right)}{c h\left[\theta_{n}(s) l\right]}\right] . \\
\vartheta_{n}(s)=\frac{l}{f E_{y}} Z(s) ; Z(s)=\frac{\operatorname{th}\left[l \theta_{n}(s)\right]}{l \theta_{n}(s)} .
\end{gathered}
$$

Here $h_{n}$ is coefficient of losses on the friction; $m, l$ are mass and length of the spring; $Z$ (s) is the spring elasticity function; $c$ is the coefficient of friction losses proportional to the stress in the mechanism [7,9].

From equations (14), (15), taking $\alpha_{n}=l \omega\left(\rho_{y} / E_{y}\right)^{1 / 2}$, one can obtain various transfer functions, for example, the function of the influence of force on fluctuations in the valve speed

$$
W_{\mathrm{v} F}(s)=\frac{\mathrm{v}_{2}(s)}{F(s)}=\frac{-\vartheta_{n}(s) s}{1-c+h_{n} \vartheta_{n}\left(\alpha_{n}\right) s+m \vartheta_{n 0}\left(\alpha_{n}\right) s^{2}} .
$$

Here $\vartheta_{n 0}$ is spring stiffness, $\vartheta_{n 0}=4 l / \pi d^{2}$.

This function in frequency form is shown in Fig. 2 for the parameters: $m=0.2 \mathrm{Ns}^{2} / \mathrm{cm}$; $\mathrm{C}=100 \mathrm{~N} / \mathrm{cm} ; d=5 \mathrm{~mm} ; l=200 \mathrm{~mm} ; D=40 \mathrm{~mm} ; D_{1}=30 \mathrm{~mm} ; z=20$ is the number of turns of the spring; $\vartheta_{n 0}=0.0131(\mathrm{Ncm})^{-1} ; h=1 \mathrm{Ns} / \mathrm{cm}$.

It is seen that at circular frequencies $\omega_{p} \simeq 19 ; 400 ; 900 \ldots \mathrm{s}^{-1}$ resonance is observed.

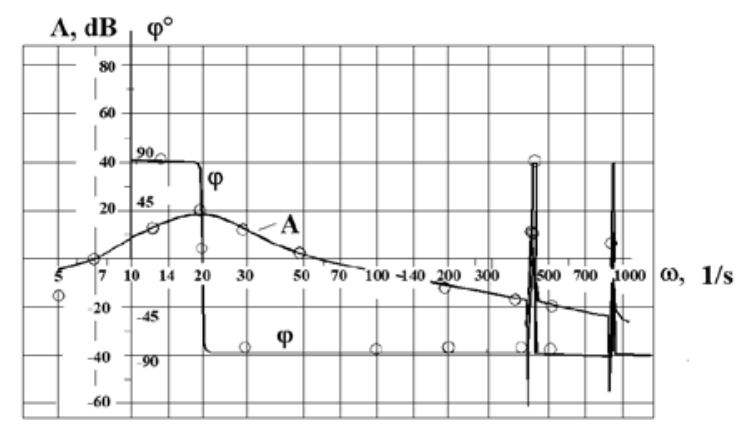

Fig. 2. Frequency characteristics of the mechanism: $A$ is amplitude; $\varphi$ is phase. 
We further assume that the influence of the distributed properties of the spring and the hysteresis phenomena on the dynamics of the body can be neglected.

Then we rewrite the equation of motion in the operator form, taking $p \equiv d / d t$

$$
(1-c) \mathrm{\cup}_{2}+h \vartheta_{n 0} p+m \vartheta_{n 0} p^{2}=(1-c) \cup_{1}-p F \vartheta_{n 0}
$$

We replaceable operator equation (18) by the system [7]

$$
\begin{gathered}
\mathrm{v}_{1}(t)=\mathrm{v}_{2}(t) \vartheta_{n 0} f p \sigma_{y}(t) \\
(1-c) \sigma_{y}(t)=F(t)+h \mathrm{v}_{2}(t)+m p \mathrm{v}_{2}(t) .
\end{gathered}
$$

Such decomposition is easily verified by an inverse algebraic solution [10]. Under the influence weight of body $P=m g$ and component $F_{1}$, friction forces $F_{m}$ develop. It is known that friction depends on the contact pressure $p_{k}$ and the relative velocity of the friction surfaces $v_{2}$. We expand the function $F_{m}\left(p_{k}, v_{2}\right)$ in a Taylor series and represent it by the expression

$$
\Delta F_{m}=\Delta p_{k} \frac{\partial F_{m}}{\partial p_{k}}+\Delta \mathrm{v}_{2} \frac{\partial F_{m}}{\partial \mathrm{v}_{2}}=c_{1} \Delta p_{k}+h \Delta \mathrm{v}_{2}
$$

The second term characterizes the losses proportional to the speed of movement. This term entered the system of equations (19) in the form of the product $h \mathrm{v}_{2}(t)$. The first term, which describes the losses on the contact area $f_{k}$, is transformed as follows

$$
c_{1} \Delta p_{k}=\frac{c_{1} F_{1}}{f_{k}}=c f \sigma_{y} .
$$

Here $c_{1}$ and $c$ are constants.

We see that this component leads to an increase in stresses of the spring. Moreover, in the case of a change in the direction of movement, the sign of this component of the force changes to the opposite. For surfaces lubricated with mineral oil with $c=0.025 \div 0.09$ [12]. In this regard, considering small deviations, we rewrite the second equation of system (19) in the form

$$
\sigma_{y} f\left(1-c \operatorname{sgn} \mathrm{v}_{2}\right)=F+h \mathrm{v}_{2}+m p \mathrm{v}_{2} .
$$

The joint solution of (19) - (22) leads to the equation

$$
\mathrm{v}_{2}\left(1-c \operatorname{sgn} \mathrm{v}_{2}\right)+h \vartheta_{n 0} p \mathrm{v}_{2}+m \vartheta_{n 0} p^{2} \mathrm{v}_{2}=\mathrm{v}_{1}\left(1-c \operatorname{sgn} \mathrm{v}_{2}\right)-p F \vartheta_{n 0} .
$$

Physical processes are well illustrated by the structural diagram in Fig. 3.

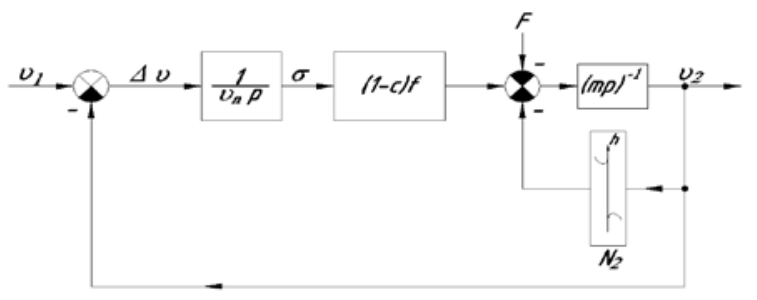

Fig. 3. Block schema of the transmission of motion in a spring-loaded mechanism. 
Here, due to the difference in the speeds of motion of the leading and driven ends, stresses develop in the spring under the action of elastic deformation, creating a driving force that overcomes of the resistance force F, friction and inertia [7].

The given formulas allow us to calculate the change in the speed of the body or stresses in a linear mechanical system [5]. In real conditions, there are always non-linearity's that often have a significant effect. In such cases, the behavior of the system can be estimated by the graph-analytical method Popov [13], or numerically analytical method using a PC.

\section{References}

1. E. Routh, The advanced part of a treatise on the dynamics of a system of rigid bodies, Macmillan and Co, London (1884)

2. A. Lyapunov, The General Problem of the Stability of Motion, (A. T. Fuller trans.), Taylor \& Francis, London (1992)

3. N. Chetaev, Stability of motion, Gostekhizdat, Moscow (1950)

4. V. Bolotin, Dynamic stability of elastic systems, Gostehizdat, Moscow (1956)

5. L. Mironova, L. Kondratenko, Method for the study of dynamic characteristics in the mechanisms of motion transmission, Journal of Vibroengineering Procedia, v. 25, pp. 214-219 (2019)

6. V. Anuryev, Directory of designer of the machine builder, v. 3. Mechanical Engineering, Moscow (1980)

7. L. Kondratenko, Calculation of vibrations in parts and components of machines, Sputnik, Moscow (2008)

8. L. Sedov, Continuum mechanics, v. 1, 2. Nedra, Moscow (1970)

9. L. Mironova, L. Kondratenko, Mathematical modeling of the processing of holes on CNC machines, Materials Today: Proceedings, v. 19(5) pp. $2354-2357$ (2019)

10. L. Mironova, L. Kondratenko, V. Terekhov, On Issue of Verifying New Method for Studying Dynamics of Deep Hole Machining, Lecture Notes in Mechanical Engineering, v. 2, pp. 151-162 (2019)

11. L. Kondratenko, L. Mironova, V. Dmitriev, Loss of stability of open two-link mechanisms. Vibroengineering Procedia, v. 25, pp. 220-225 (2019)

12. V. Prokofiev, Axial piston adjustable hydraulic drive, Mashinostroenie, Moscow (1969)

13. E. Popov, Applied theory of control processes in nonlinear systems, FML, Moscow (1973) 